\title{
Equation of state for chain molecules: Continuous-space analog of Flory theory
}

\author{
Ronald Dickman and Carol K. Hall \\ Department of Chemical Engineering, North Carolina State University, Raleigh, North Carolina \\ 27695-7905
}

(Received 29 May 1986; accepted 16 June 1986)

\begin{abstract}
New, accurate equations of state for fluids of chain molecules are derived as generalizations of the well-known Flory and Flory-Huggins lattice theories to continuous space. Comparison with the results of new Monte Carlo simulations of athermal chains (freely jointed hard disks and spheres), extending over a wide range of densities, reveals that the generalized FloryHuggins equation of state provides an accurate prediction for the pressure.
\end{abstract}

\section{INTRODUCTION}

Fluids composed of chain molecules are of increasing interest, owing to their richly varied static and dynamic properties, and to their obvious importance in the natural gas and petroleum industries. While the techniques commonly employed in theories of simple liquids (integral equations, perturbation theory) have been extended to molecular fluids, ${ }^{1}$ such approaches often pose formidable computational difficulties when applied to chain molecules. In view of these difficulties, and of the relatively primitive level of understanding of such fluids, the development of simple mean field theories for estimating their properties is desirable. Two mean field theories-the Flory $(F)$ and FloryHuggins (FH) approximations, ${ }^{2,3}$-have, since their introduction over forty years ago, found wide application in the statistical mechanics of polymer solutions ${ }^{4-6}$ and mixtures. ${ }^{7}$ In this paper we develop accurate equations of state, based on the Flory and Flory-Huggins approaches. We shall show that quantitative predictions for the pressure may be derived, provided that (1) the equation of state for a fluid comprised of the individual chain elements (monomers) is known to good approximation, and (2) the fact that real fluids exist in continuous space (as opposed to a lattice) is properly accounted for. The resulting equations of state are simple in form and are in excellent agreement with simulation results for athermal chains.

The original $\mathrm{F}$ and $\mathrm{FH}$ arguments appealed to a lattice picture for estimating the number of configurations, but certain of the resulting expressions (for example, the entropy of mixing), are free of lattice-related parameters, and would appear to be applicable to continuous-space systems. One may interpret the lattice coordination number as representing the average number of neighbors in a fluid, thereby eliminating from the final results any overt reference to the lattice picture. Nevertheless, certain predictions of the $\mathrm{F}$ and $\mathrm{FH}$ theories-in particular the equation of state-betray, in their basic structure, their discrete-space provenance. For chains on lattices, detailed comparisons with simulation results $^{8-11}$ have shown that the mean field theory predictions for the osmotic pressure are rather accurate at moderate to high densities. However, it has recently become apparent that direct application of the lattice formulas yields severe underestimates for the pressure in continuous-space models. ${ }^{12,17}$ In this paper we shall uncover the reason for this discrepancy, and then show how the probabilistic assumptions employed in the lattice model derivations may be taken over to a continuous-space context to yield accurate equations of state. The predictions of the continuous-space versions of mean field theory will then be compared with results of new Monte Carlo simulations of athermal chains. The latter are also of intrinsic interest, since our studies extend to higher densities than have been investigated before.

We begin, in Sec. II, by reviewing the derivations of $F$ and FH theories for chains on a lattice. By casting the argument in terms of simple, precise probability concepts, we are able to pinpoint the assumptions and approximations required. The application of analogous assumptions and approximations to continuous-space models is explored in Sec. III, leading to what may be termed "generalized" $F$ and FH equations of state. In Sec. IV we describe our Monte Carlo simulation method, and compare the results with the predictions of the generalized mean field theories.

\section{LATTICE MODEL}

We consider chains of $n$ segments on a lattice, which interact via a pairwise segment-segment interaction. The intramolecular contribution to the potential energy may also depend on the relative orientation of successive bonds. We write the total potential energy of a system of $N_{p}$ chains as

$$
U_{N_{p}}=\frac{1}{2} \sum_{i \neq j} \sum_{k, l=1}^{n} u\left(\left|x_{i}^{(k)}-x_{j}^{(l)}\right|\right)+\sum_{i=1}^{M} w\left(x_{i}^{(1)}, \ldots, x_{i}^{(n)}\right),
$$

where $x_{i}^{(k)}$ denotes the position of the $k$ th segment of the $i$ th chain. We shall assume that $u=u_{0}+u^{\prime}$ and $v=v_{0}+v^{\prime}$, where $u_{0}$ and $v_{0}$ are site-exclusion interactions which assign infinite energy to any configuration in which a site is occupied by two or more segments. The partition function for a system of $N_{p} n$-mers on a lattice of $N$ sites is

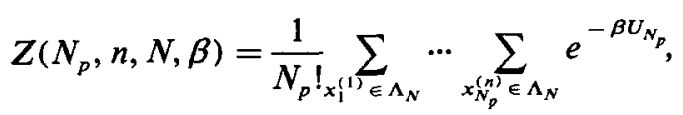

where $\Lambda_{N}$ is a region of the lattice encompassing $N$ sites, and $\beta=1 / k_{B} T$.

Let $Y$ denote an $n$-mer configuration, and let $X$ denote a configuration of $N_{p} n$-mers on a lattice of $N$ sites. A quantity 
of key interest is the insertion factor

$$
p\left(N_{p}, n, N, \beta\right)=\left\langle\left\langle e^{-\beta U_{T}}\right\rangle_{Y}\right\rangle_{X}
$$

where $U_{T}$ is the potential energy of "test-chain" configuration $Y$, when inserted into the many-chain configuration $X$. The brackets indicate ensemble averages over the system and test-chain configurations. Referring to Eq. (2.2), we have $p\left(N_{p}, n, N, \beta\right)$

$$
\begin{aligned}
& =\frac{\Sigma_{x_{1}^{(1)} \cdots \Sigma_{x_{N_{p}+1}^{(n)}}} e^{-\beta U_{N_{p}+1}}}{\left[\Sigma_{x_{1}^{(1)}} \cdots \Sigma_{x_{N_{p}}^{(n)}} e^{-\beta U_{N_{p}}}\right]\left[\Sigma_{x_{1}^{(1)}} \cdots \Sigma_{x_{1}^{(n)}} e^{-\beta U_{1}}\right]} \\
& =\left(N_{p}+1\right) \frac{Z\left(N_{p}+1, n, N, \beta\right)}{Z\left(N_{p}, n, N, \beta\right) Z(1, n, N, \beta)} .
\end{aligned}
$$

The partition function may therefore be written as

$$
Z\left(N_{p}, n, N, \beta\right)=\frac{[Z(1, n, N, \beta)]^{N_{p}}}{N_{p} !} \prod_{j=1}^{N_{p}-1} p(j, n, N, \beta) .
$$

Using $\pi$ and $\mu$ to denote, respectively, the pressure and chemical potential of the chains, we have the thermodynamic relation

$$
\mu N_{p}=E-T S+\pi N .
$$

If we use the relations

$$
A\left(N_{p}, n, N, \beta\right)=E-T S=-k_{B} T \ln Z\left(N_{p}, n, N, \beta\right)
$$

and

$$
\begin{aligned}
\mu\left(N_{p}, n, N, \beta\right)= & -k_{B} T\left[\ln Z\left(N_{p}+1, n, N, \beta\right)\right. \\
& \left.-\ln Z\left(N_{p}, n, N, \beta\right)\right]
\end{aligned}
$$

together with Eq. (2.5), then we have

$$
\begin{aligned}
\pi^{*}\left(N_{p}, n, N, \beta\right) \\
=N^{-1}\left[\sum_{j=1}^{N_{p}-1} \ln p(j, n, N, \beta)-N_{p} \ln p\left(N_{p}, n, N, \beta\right)\right. \\
\left.\quad+N_{p} \ln \left(N_{p}+1\right)-\ln N_{p} !\right],
\end{aligned}
$$

where $\pi^{*} \equiv \beta \pi$. factor

We shall assume the existence of the limiting insertion

$$
p(\phi, n, \beta) \equiv \lim _{N, N_{p} \rightarrow \infty ; n N_{p} / N=\phi} p\left(N_{p}, n, N, \beta\right) .
$$

Taking the limit $N, N_{p} \rightarrow \infty$, with fixed occupation fraction $\phi$, Eq. (2.9) becomes the osmotic equation of state

$\pi^{*}(\phi, n, \beta)$

$$
=\frac{\phi}{n}[1-\ln p(\phi, n, \beta)]+\frac{1}{n} \int_{0}^{\phi} \ln p\left(\phi^{\prime}, n, \beta\right) d \phi^{\prime} .
$$

We now specialize to the case $u^{\prime}=v^{\prime}=0$, i.e., fully flexible, athermal chains on a lattice. Since the only interactions are site-exclusion, $e^{-\beta U}=1$ if the configuration is free of overlap, and is zero otherwise. Thus $p\left(N_{p}, n, N\right)$ (we drop the argument $\beta$ since $p$ is now temperature independent) may be interpreted as the probability that a randomly chosen $n$-mer may be inserted without overlap into a randomly chosen configuration of $N_{p} n$-mers on a lattice of $N$ sites. The Flory (F) and Flory-Huggins (FH) theories provide simple estimates for $p(\phi, n)$ for athermal lattice chains. We shall review the F and FH derivations, so as to highlight the approximations involved, and to facilitate their extension to off-lattice models in the following section.

Let $\chi(Y, X)$ be 1 if chain $Y$ does not overlap with any of the $N_{p}$ chains in configuration $X$, and let $\chi$ be zero otherwise. Then we may express the insertion probability as an average over configurations:

$$
p\left(N_{p}, n, N\right)=\left\langle\langle\chi(Y, X)\rangle_{Y}\right\rangle_{X},
$$

where averages over all the $Y$ and $X$ are indicated. We may also define

$$
P_{N_{p, n}, N}(Y)=\langle\chi(Y, X)\rangle_{X}
$$

which is the probability that $n$-mer configuration $Y$ may be inserted without overlap into a randomly chosen configuration of $N_{p} n$-mers, so that $p\left(N_{p}, n, N\right)=\left\langle P_{N_{p, n, N}}(Y)\right\rangle_{Y}$.

For $y_{i}, y_{j}$, etc, $\in \Lambda_{N}$, define $P_{N_{p}, n, N}\left(y_{i} \mid y_{k}, \ldots, y_{m}\right)$ as the probability that $y_{i}$ is vacant in a randomly chosen configuration of $N_{p} n$-mers on a lattice of $N$ sites, given that sites $y_{k}, \ldots, y_{m}$ are vacant. Let $Y=\left(y_{1}, y_{2}, \ldots, y_{n}\right)$, where the sites are listed in their order along the chain. Then

$$
\begin{aligned}
P_{N_{p, n, N}}(Y)= & (1-\phi) P_{N_{p, n, N}}\left(y_{2} \mid y_{1}\right) P_{N_{p, n, N}}\left(y_{3} \mid y_{1}, y_{2}\right) \\
& \times \cdots \times P_{N_{p, n, N}}\left(y_{n} \mid y_{1}, \ldots, y_{n-1}\right) .
\end{aligned}
$$

In the Flory approximation sites $y_{1}, \ldots, y_{n}$ are regarded as occupied independently with probability $\phi$, so that for $k>2$,

$$
P_{N_{p, n, N}}\left(y_{k} \mid y_{1}, \ldots, y_{k-1}\right)=(1-\phi),
$$

which immediately yields

$$
p_{\mathrm{F}}(\phi, n)=(1-\phi)^{n} \text {. }
$$

The Flory approximation treats the occupied sites as if they were scattered randomly over the lattice, rather than grouped in chains.

To derive the FH formula for $p$, we first invoke a Markovian assumption

$$
P_{N_{p, n, N}}\left(y_{k} \mid y_{1}, \ldots, y_{k-1}\right)=P_{N_{p, n, N}}\left(y_{k} \mid y_{k-1}\right)
$$

for $k \geqslant 2$. As a result of this approximation, the insertion probability

$$
P_{N_{p, n, N}}(Y)=(1-\phi)\left[P_{N_{p, n, N}}\left(y \mid y^{\prime}\right)\right]^{n-1}
$$

is independent of $Y$, since $y$ and $y^{\prime}$ are an arbitrary pair of neighboring sites. Thus $p_{\mathrm{FH}}\left(N_{p}, n, N\right)=(1-\phi)\left(1-\phi_{0}\right)^{n-1}$, where $\phi_{0}$ is the probability that a neighbor of a randomly chosen vacant site is occupied. While this represents a considerable simplification over Eq. (2.14), determination of $\phi_{0}$ is not trivial, since knowledge of the pair correlation for vacant sites is required. In the FH theory $\phi_{0}$ is approximated as the occupation probability $\widetilde{\phi}$ of a neighbor of any site (occupied or vacant), excluding pairs of sites occupied by successive segments of a chain. The accuracy of this approximation is difficult to assess, but it clearly represents an improvement over the complete disregard for chain structure inherent in the Flory approximation. $\bar{\phi}$ may be evaluated as follows. If a fraction $\phi$ of the sites are occupied by chain segments, then $N \phi(n-2) / n$ sites are internal to chains. Denoting the lattice coordination number by $z$, the density of occupied neigh- 
bors of the internal sites is

$$
\phi_{\mathrm{in}}=\frac{z-2}{z} \widetilde{\phi}+\frac{2}{z},
$$

since each internal site has two neighbors which are surely occupied. Similarly, neighbors of the $2 N \phi / n$ chain-end sites are occupied with probability

$$
\phi_{\text {end }}=\frac{z-1}{z} \tilde{\phi}+\frac{1}{z}
$$

and, by assumption, neighbors of the $N(1-\phi)$ vacant sites are occupied with probability $\widetilde{\phi}$. Since the overall fraction of occupied sites is $\phi$, we must have

$$
\phi=\phi\left[\left(1-\frac{2}{n}\right) \phi_{\text {in }}+\frac{2}{n} \phi_{\text {end }}\right]+(1-\phi) \widetilde{\phi},
$$

which implies that

$$
\widetilde{\phi}=\phi \frac{1-2 z^{-1}\left(1-n^{-1}\right)}{1-2 \phi z^{-1}\left(1-n^{-1}\right)} .
$$

The insertion probability in the FH approximation is therefore

$$
\begin{aligned}
p_{\mathrm{FH}}(\phi, n) & =(1-\phi)(1-\widetilde{\phi})^{n-1} \\
& =(1-\phi)^{n}\left[1-2 \phi z^{-1}\left(1-n^{-1}\right)\right]^{-(n-1)} .
\end{aligned}
$$

Numerical studies ${ }^{8-11}$ indicate that at low and moderate densities the $\mathrm{F}$ and $\mathrm{FH}$ formulas tend to underestimate the insertion probability. However, the FH estimate for the pressure is quite accurate over a wide range of densities. The disparity between $\mathrm{F}$ and $\mathrm{FH}$ predictions and numerical results worsens with increasing chain length, which is not surprising, given that (1) the $\mathrm{F}$ and $\mathrm{FH}$ theories are exact for $n=1$, and (2) correlations within and between chains (ignored in these theories) assume greater importance for longer chains.

\section{EXTENSION TO OFF-LATTICE MODELS}

In the theory of athermal polymers and mixtures, the $F$ and $\mathrm{FH}$ approximations, originally derived for lattice models, are frequently applied to off-lattice models. ${ }^{4-7}$ The transition from lattice to continuous space is accomplished by replacing the site occupation fraction $\phi$ appearing in Eqs. (2.16) and (2.23) with $\eta$, the fraction of the total volume occupied by molecules. (In the case of chains composed of $n$ tangent hard spheres of diameter $\sigma, \eta=n \pi \sigma^{3} \rho_{n} / 6$, where $\rho_{n}=N_{p} / V$ is the density of $n$-mers). However, recent studies $^{12,17}$ reveal that such an approach yields a severe underestimate for the pressure. As the following discussion makes clear, the lattice formulas are fundamentally inappropriate for describing continuous-space systems. However, it is not difficult to extend the $\mathrm{F}$ and $\mathrm{FH}$ approaches to a continuousspace context, and to derive expressions analogous to Eqs. (2.16) and (2.23), which provide accurate predictions for the pressure in a system of athermal chains.

Let $Z\left(N_{p}, n, V, \beta\right)$ be the partition function for a system of $N_{p} n$-mers in volume $V$, and let $p\left(N_{p}, n, V, \beta\right)$ be the insertion factor, i.e., Eq. (2.3) with $N$ replaced by $V$. The relation between $p$ and $Z$ is again given by Eq. (2.4), if we replace $N$ by $V$. In analogy with Eq. (2.10), we assume the existence of

$$
p(\eta, n, \beta) \equiv \lim _{N_{p}, V \rightarrow \infty ; N_{p} v_{n} / V=\eta} p\left(N_{p}, n, V, \beta\right),
$$

where $v_{n}$ is the volume of an $n$-mer. If $N$ is replaced by $V$, Eq. (2.9) also holds for continuous-space systems, and in the thermodynamic limit the continuous-space version of the osmotic equation of state is

$$
\begin{aligned}
\pi^{*}(\eta, n, \beta)= & \frac{\eta}{v_{n}}[1-\ln p(\eta, n, \beta)] \\
& +\frac{1}{v_{n}} \int_{0}^{\eta} \ln p\left(\eta^{\prime}, n, \beta\right) d \eta^{\prime} .
\end{aligned}
$$

Comparing this with Eq. (2.11), one may be tempted to conclude that the continuous-space system is described by the lattice formula, with $\phi \rightarrow \eta$ and $n \rightarrow v_{n}$. However, it will be seen presently that $p_{\text {continuum }}(\eta) \neq p_{\text {lattice }}(\eta)$. In fact, the dependence of the insertion probabilities on the volume fraction is radically different in the two cases.

To begin, we consider the case of athermal chains with $n=1$, for which the lattice $\mathrm{F}$ and $\mathrm{FH}$ theories both give $p(\phi, 1)=(1-\phi)$, which is exact. In this case, Eq. (2.9) yields the familiar result

$$
\pi^{*}(\phi, 1)=-\ln (1-\phi) .
$$

For arbitrary $n$, the Flory approximation predicts the compressibility factor

$$
\rho_{n}^{-1} \pi_{\mathrm{F}}^{*}(\phi, n)=1-\frac{n}{\phi}[\ln (1-\phi)+\phi],
$$

where $\rho_{n}$ is the number of chains per unit volume. Equation (3.4) (with $\phi$ replaced by $\eta$ ) is commonly cited as the Flory theory prediction for the compressibility factor in a system of athermal chains in continuous space. Since, on the lattice, the $\mathrm{F}$ and $\mathrm{FH}$ theories are exact for $n=1$ and only approximate for $n>1$, we should not expect Eq. (3.4) to be a good approximation for the continuum unless the pressure in a system of monomers (e.g., hard spheres) is well-approximated by $-\ln (1-\eta)$. But this is obviously a gross underestimate of the pressure. For example, the pressure in the fluid phase of hard spheres is accurately described by the Carnahan-Starling equation ${ }^{13}$

$$
\pi_{\mathrm{CS}}^{*}(\eta)=\rho \frac{1+\eta+\eta^{2}-\eta^{3}}{(1-\eta)^{3}}
$$

while in the case of hard rods of length $\sigma$ on a line, we have the exact result ${ }^{14}$

$$
\pi_{\text {rods }}^{*}(\eta)=\frac{\rho}{1-\eta},
$$

where $\eta=\sigma \rho$. We see that as the volume fraction approaches unity, the pressure increases much more rapidly in continuous-space models than on the lattice. (The same conclusion applies to the FH lattice approximation, which predicts a compressibility factor somewhat smaller than in the $F$ approximation.)

We shall now derive generalizations of the $\mathrm{F}$ and $\mathrm{FH}$ theories for athermal chains in continuous space, by arguments which parallel the lattice model derivations of the previous section. In the lattice model derivations, estimation of $p(\phi, n)$ was based on the knowledge of the monomer inser- 
tion probability $p(\phi, 1)=(1-\phi)$. It is not possible, in general, to derive an exact expression for the monomer insertion probability $\rho(\eta, 1)$, for a continuous-space model. However, from Eq. (3.2) we have

$$
\ln p(\eta, 1)=\int_{0}^{\eta} \frac{d \eta^{\prime}}{\eta^{\prime}}\left[1-v_{1} \frac{d \pi^{*}\left(\eta^{\prime}, 1\right)}{d \eta^{\prime}}\right]
$$

so that, given an approximate equation of state for monomers, we can derive an estimate for the monomer insertion probability. One case for which an exact evaluation is possible is the hard rod system; inserting Eq. (3.6) into the above expression we find

$$
p_{\text {rods }}(\eta ; 1)=(1-\eta) \mathrm{e}^{-\eta /(1-\eta)} \text {. }
$$

As $\eta$ approaches unity, the insertion probability for rods vanishes much more rapidly than the corresponding lattice probability. The reason is that most of the volume fraction $1-\eta$ not occupied by rods occurs in segments shorter than $\sigma$, and is therefore unavailable for occupancy by additional rods. The monomer insertion probability for hard spheres, derived from the Carnahan-Starling equation (3.5), is readily found to be

$$
p_{\mathrm{CS}}(\eta, 1)=\exp \left[-\frac{\eta\left(8-9 \eta+3 \eta^{2}\right)}{(1-\eta)^{3}}\right]
$$

which again decays rapidly with increasing $\eta$.

In deriving the Flory formula for the lattice model, the $n$-mer insertion probability was taken to be $[p(\phi, 1)]^{n}$, which follows from the assumption that sites are occupied independently and with equal probability. To extend this approach to athermal continuous-space models, we introduce the concept of the exclusion region associated with an $n$-mer. We present the argument for chains composed of freely jointed, nonoverlapping tangent hard spheres. Other molecular models may be treated in an analogous manner.

In a fluid composed of hard sphere molecules (monomers) of diameter $\sigma$, if a molecule is centered at $x$, then no other monomers may have their centers within the sphere of radius $\sigma$, centered at $x$. The exclusion volume associated with a monomer is $v_{e}(1)=4 \pi \sigma^{3} / 3$, and $p(\eta, 1)$ is the probability that a sphere of volume $v_{e}(1)$, placed into the fluid at random, is free of centers of any molecules. Let $p_{1}(\eta, n)$ be the probability that a sphere of volume $v_{e}(1)$, inserted at random into a fluid of hard-sphere $n$-mers is free of centers of any chain segments, i.e., $p_{1}(\eta, n)$ is the probability for insertion of a single spherical segment into the $n$-mer fluid. We shall approximate $p_{1}(\eta, n)$ by $p(\eta, 1)$, the insertion probability for the monomer fluid. With each $n$-mer configuration $Y$ we may associate an exclusion region: the region of space which must be free of centers of monomers, if insertion of $Y$ is possible. The insertion probability $P(Y)$ is the probability of finding the corresponding exclusion region, centered at a randomly chosen point in the fluid, free of centers of monomers. Clearly, an exact evaluation of $P(Y)$ would require knowledge of the probability distribution for voids in the fluid. In the absence of detailed information regarding the distribution of voids, we shall assume that $P(Y)$ is a function of the exclusion volume $v_{e}(Y)$ only. As in the Flory lattice argument, we shall also assume that the vacancies of disjoint regions constitute independent events. Under these assumptions, the insertion probability becomes

$$
P_{\mathrm{F}}(Y)=[p(\eta, 1)]^{v_{e}(Y) / v_{e}(1)}
$$

and therefore,

$$
p_{\mathrm{F}}(\eta, n)=[p(\eta, 1)]^{v_{e}(n) / v_{e}(1)},
$$

where $v_{e}(n)$ is the $n$-mer exclusion volume, and the subscript $F$ denotes the extension of the Flory-type argument to continuous space.

It is important to note that in general, $v_{e}(n) \neq n v_{e}(1)$. For example, in the case of hard spheres, the overlap between exclusion regions associated with neighboring monomers is such that

$$
v_{e}(2)=\frac{9}{4} \pi \sigma^{3}=\frac{27}{16} v_{e}(1) .
$$

For $n \geqslant 3, v_{e}(Y)$ may depend on the particular choice of $Y$; $v_{e}(n)$ is then the exclusion volume averaged over all $n$-mer configurations. The evaluation of $v_{e}(n)$ for chains of freely jointed hard spheres and disks is considered in the Appendix.

Inserting Eq. (3.11) into the equation of state, Eq. (3.2), we find

$$
\begin{aligned}
\pi_{\mathrm{F}}^{*}(\eta, n)= & \frac{\eta}{v_{n}}\left[1-\frac{v_{e}(n)}{v_{e}(1)} \ln p(\eta, 1)\right] \\
& +\frac{v_{e}(n)}{v_{e}(1) v_{n}} \int_{0}^{\eta} \ln p\left(\eta^{\prime}, 1\right) d \eta^{\prime} \\
= & \lambda_{n} \pi^{*}(\eta, 1)+\rho_{n}\left(1-\frac{v_{n} \lambda_{n}}{v_{1}}\right),
\end{aligned}
$$

where $\lambda_{n} \equiv v_{1} v_{e}(n) / v_{n} v_{e}$ (1). Equations (3.11) and (3.13) constitute the extension of Flory theory to a continuousspace system of athermal chains. The dependence of $\pi_{\mathrm{F}}^{*}$ on $\eta$ is inherited from the monomer equation of state, and so we should not expect the severe underestimate of the pressure inherent in the lattice formulas, Eqs. (3.3) and (3.4).

Our generalization of the FH approach to continuousspace models is based on the idea that the volume fraction $\widetilde{\eta}$ in the neighborhood of a void large enough to accommodate a monomer, is smaller than the average volume fraction $\eta$, because monomers are grouped together in chains. Thus the FH estimate for the insertion probability is

$$
p_{\mathrm{FH}}(\eta, n)=p(\eta, 1)[p(\widetilde{\eta}, 1)]^{v_{e}(n) / v_{e}(1)-1} .
$$

In deriving $\widetilde{\eta}$ for continuous-space models, we may follow the chain of reasoning employed in Eqs. (2.17)-(2.23), the sole modification being that the lattice coordination number $z$ is replaced by $\bar{z}$, the average coordination number at close packing. Since we are considering a disordered phase, the coordination number characteristic of random close packing is appropriate: $\bar{z}=9$ for hard spheres, ${ }^{15}$ and $\bar{z}=5$ for hard disks. ${ }^{16}$ By analogy with Eq. (2.22),

$$
\tilde{\eta}=\eta \frac{1-2 \bar{z}^{-1}\left(1-n^{-1}\right)}{1-2 \eta \bar{z}^{-1}\left(1-n^{-1}\right)}=\frac{\eta(1-\alpha)}{1-\alpha \eta},
$$

where $\alpha \equiv 2 \bar{z}^{-1}\left(1-n^{-1}\right)$.

Our generalization of the FH approximation to contin- 
uous-space systems yields the equation of state

$$
\begin{aligned}
\pi_{\mathbf{F H}}^{*}(\eta, n)= & \frac{v_{1}}{v_{n}} \pi^{*}(\eta, 1)-\left(\frac{\lambda_{n}}{v_{1}}-\frac{1}{v_{n}}\right) \\
& \times\left[\eta \ln p(\tilde{\eta}, 1)-\int_{0}^{\eta} \ln p\left(\widetilde{\eta}^{\prime}, 1\right) d \eta^{\prime}\right],
\end{aligned}
$$

where $\widetilde{\eta}^{\prime}$ is given by Eq. (3.15) with $\eta^{\prime}$ in place of $\eta$. Using Eqs. (3.7) and (3.15), and integrating the last term by parts, one finds

$$
\begin{aligned}
\pi_{\mathrm{FH}}^{*}(\eta, n)= & \frac{v_{1}}{v_{n}} \pi^{*}(\eta, 1)-\left(\frac{\lambda_{n}}{v_{1}}-\frac{1}{v_{n}}\right) \int_{0}^{\eta} \frac{d \eta^{\prime}}{1-\alpha \eta^{\prime}} \\
& \times\left[1-\left.v_{1} \frac{d \pi^{*}(y ; 1)}{d y}\right|_{y=(1-\alpha) \eta^{\prime} / 1-\alpha \eta^{\prime}}\right]
\end{aligned}
$$

for the pressure in the generalized $\mathrm{FH}$ approximation. If we take $\pi^{*}(\eta, 1)$ to be the Carnahan-Starling equation (3.5), the above expression yields

$$
\begin{aligned}
\pi_{\mathrm{FH}-\mathrm{CS}}^{*} & (\eta, n) \\
= & n^{-1} \pi_{\mathrm{CS}}^{*}(\eta, 1)+v_{n}^{-1}\left(\frac{v_{e}(n)}{v_{e}(1)}-1\right)(1-\alpha) \\
& \times\left(2 \alpha(1+3 \alpha) \ln (1-\eta)+\frac{\eta}{(1-\eta)^{3}}\right. \\
& \times\{2 \alpha(1+3 \alpha)+[4-5 \alpha(1+3 \alpha)] \eta \\
& \left.\left.-\left(2+\alpha-11 \alpha^{2}\right) \eta^{2}\right\}\right) .
\end{aligned}
$$

The generalized $\mathrm{F}$ and $\mathrm{FH}$ theories will be compared against other theories and simulation results in the following section.

While Eqs. (3.13) and (3.17) have been derived for athermal models, the following argument suggests that they are valid for more general interactions. Consider models in which the intermolecular potential $u$ includes a steep repulsion (e.g., hard-core square-well or Lennard-Jones potentials), with which a hard-core diameter $\sigma_{0}$ may be associated. Then we may take $v_{1}=\pi \sigma_{0}^{3} / 6$ and $\eta=n \rho_{n} v_{1}$, so that the parameters in Eq. (3.17) are well defined. [Of course, $\pi^{*}(\eta, 1, \beta)$ is now the pressure in a monatomic system with interparticle potential $u$.] For those configurations which make the dominant contribution to $p(\eta, n, \beta)$ (i.e., those for which $U_{T}$ is small), the presence of a test-chain segment effectively excludes segments of other chains from a sphere of radius $\sigma_{0}$. The argument based on the overlap of exclusion regions of successive segments along a chain therefore remains valid, and so we may expect Eqs. (3.13) and (3.17) to be applicable to nonathermal models with an effective hard core.

\section{MONTE CARLO SIMULATIONS}

In order to test the generalized $\mathrm{F}$ and $\mathrm{FH}$ theories derived above, it is desirable to compare their predictions with the results of simulations of athermal chains. We therefore undertook a series of Monte Carlo simulations with the aim of estimating the pressure for as wide a range of densities as possible.

In our simulations we studied athermal chains composed of $n$ freely jointed, nonoverlapping tangent disks (in two dimensions) and spheres (in three dimensions), of unit diameter. The simulation cell was a square or cube with periodic boundary conditions. In a straightforward application of the Metropolis algorithm ${ }^{18}$ to an athermal system, a trial configuration is accepted if and only if it is free of overlap. The configuration of the $i$ th chain is specified by the position $x_{i}^{(1)}$ of the center of the first element, and a set of unit vectors $e_{i}^{(1)}, \ldots, e_{i}^{(n-1)}, \quad\left(e_{i}^{(j)}=x_{i}^{(j+1)}-x_{i}^{(j)}\right)$. A trial configuration is generated by subjecting $x_{i}^{(1)}$ and the $e_{i}^{(j)}$ (for some $i$ chosen at random) to random displacements, with the $\left|e_{i}^{(j)}\right|$ held fixed. (The magnitudes of the random displacements were adjusted so as to achieve an acceptance rate of $30 \%-50 \%$.)

As in our simulations of lattice chains, ${ }^{11}$ we sample the configuration space of $N_{p} n$-mers in volume V (the "system"), as well as the configurations of an isolated test chain. (Each step in the simulation involves the generation of a trial main configuration and an independent trial configuration for the test chain.) At each step it is determined whether the test chain would overlap with any of the chains of the main configuration, were it to be inserted at random into the simulation cell. An unbiased estimator for the insertion probability $p\left(N_{p}, n, V\right)$ is the average rate of overlap-free insertion of the test chain. Estimates for $p\left(N_{p}, n, V\right)$ were derived by averaging the insertion rate over 10 runs, each consisting of between $5 \times 10^{4}$ and $3 \times 10^{5}$ steps, the first $10 \%$ of which were discarded in order to allow for relaxation.

In Fig. 1, the solid circles show a possible configuration of the system. The volume fraction $\eta$ is the fraction of the system area which lies within the disks. (For chains composed of $n$ tangent hard disks of unit diameter, $\eta=n \pi N_{p} /$

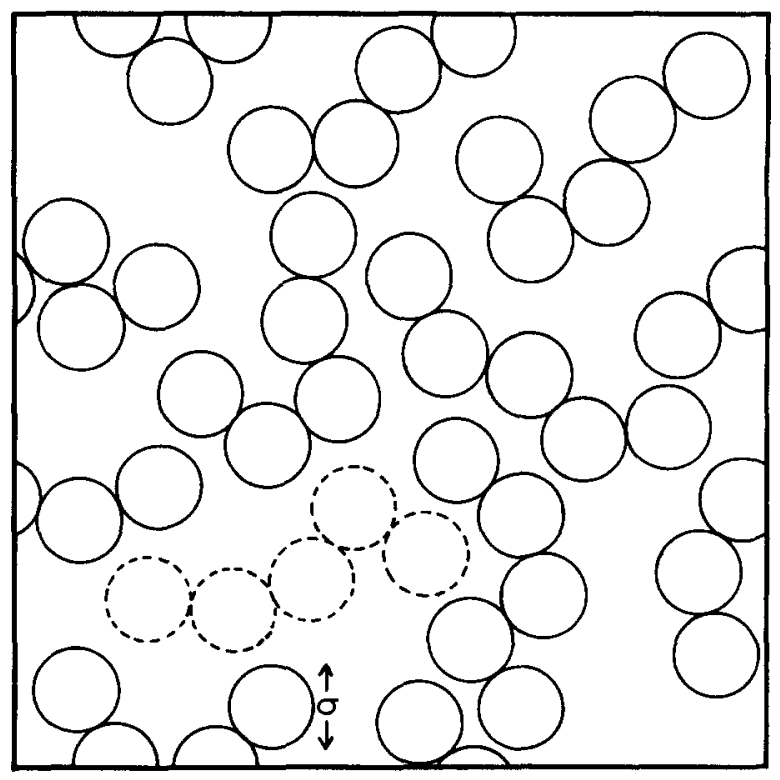

FIG. 1. A possible system configuration (solid circles) in the Monte Carlo simulation of hard-disk chains. The broken circles indicate a possible insertion of the test chain. 


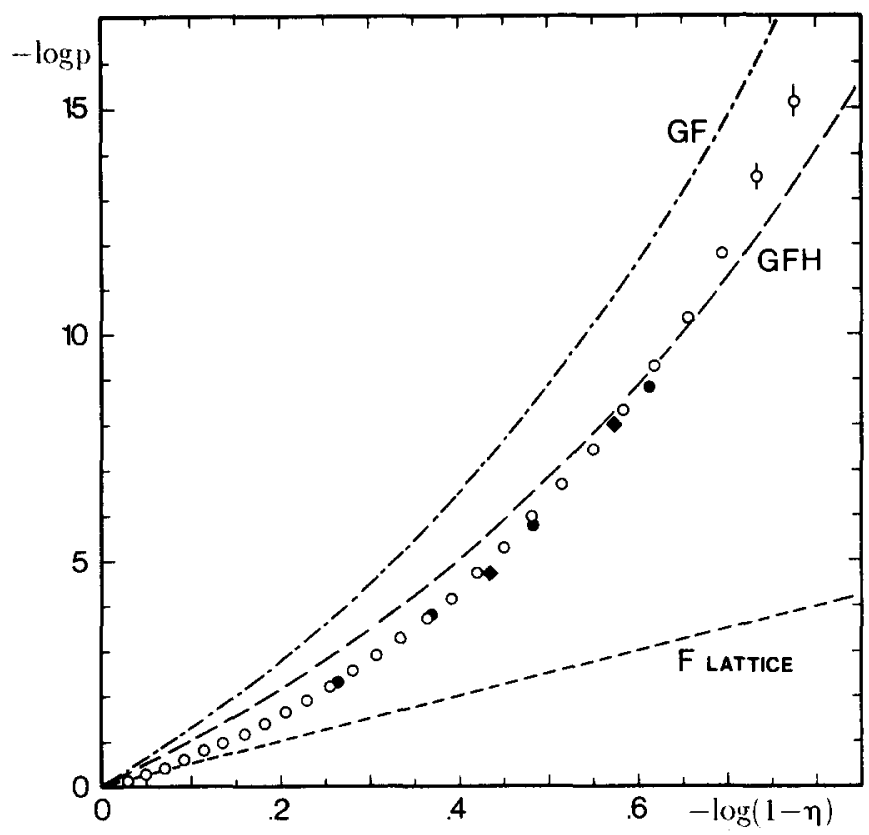

FIG. 2. Monte Carlo estimates for $\ln p(\eta, n)$ vs $\ln (1-\eta)$ for chains of 5 hard disks. Open circles: $L=20$; filled circles: $L=25$; diamonds: $L=30$. The curves represent predictions of Flory lattice theory, and the generalized Flory (GF) and generalized Flory-Huggins (GFH) theories.

$4 A$, where $A$ is the total area; $\eta$ is related to the monomer density $\rho$ through $\eta=0.9069 \rho / \rho_{c p}$, where $\rho_{c p}=1.1547$ is the close-packing density for disks of unit diameter.) A possible insertion of the test chain (broken circles) is also depicted. The insertion probability $p(\eta, n)$ is the probability, averaged over all system and test-chain configurations, that the test chain may be inserted into the system without overlapping with any of the existing chains.

To test the mean field theory predictions, we require an estimate of the limiting insertion probability $p(\eta, n)$. In the course of adding a new chain to a finite system, the volume fraction grows from $v_{n} N_{p} / V$ to $v_{n}\left(N_{p}+1\right) / V$, and so we regard the finite-size insertion probability $p\left(n_{p}, n, V\right)$, as an estimate for $p(\eta ; n)$ at the mean volume fraction $\eta=v_{n}\left(N_{p}+\frac{1}{2}\right) / V$. Our simulations of hard-disk chains were carried out on a square cell of length $L / \sqrt{ } 2$, with $L=20$ in most cases. (The disks were of unit diameter.) As a test for finite-size effects several simulations were performed with $L=25$ and $L=30$. Our estimates for $\ln p(\eta ; n)$ for chains composed of five tangent hard disks are plotted vs $\ln (1-\eta)$ in Fig. 2. For $\eta<0.3$, the $L=20$ and $L=25$ estimates agree to within uncertainty, while for $\eta$ in the range $0.38-0.45$, the $L=20$ estimates for $\ln p(\eta, n)$ are about $3 \%$ smaller than the $L=25$ estimates. For $\eta=0.44$, the $L=20$ and $L=30$ estimates do not differ by more than $3 \%$, and so the difference between $\ln p(\eta ; n)$ and the $L=20$ estimate is not likely to exceed 3\%-4\%. In light of Eq. (3.2), we may therefore expect estimates for the pressure based on the $L=20$ data to be a few percent too large for $\eta>0.38$. [Note that there will be a partial cancellation of the error in the pressure, since $\ln p$ appears with opposite sign in the first and second terms of Eq. (3.2)].

The curves in Fig. 2 represent the predictions of the $F$ lattice formula, Eq. (2.16), and of our generalized F and FH

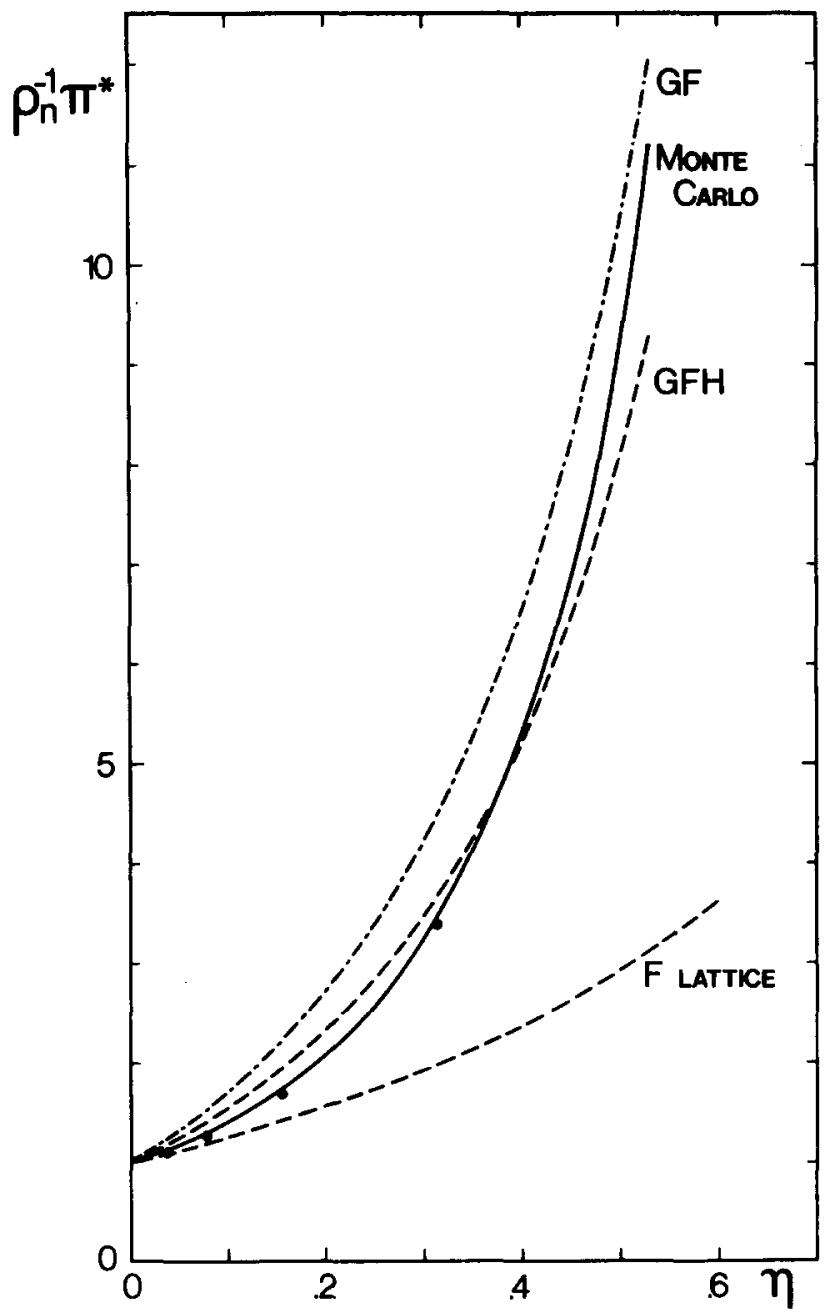

FIG. 3. Comparison of the compressibility factor $\rho_{n}^{-1} \pi^{*}$, vs $\eta$ for chains of 5 hard disks, as obtained from Monte Carlo simulations, with predictions of the Flory lattice formula, and generalized Flory (GF) and Flory-Huggins (GFH) theories. The circles are Monte Carlo estimates derived by Okamoto (Ref. 17) for the pressure in a system of hard-disk chains $(n=5)$ in which bond angles are restricted to the values: $\pi, \pm \pi / 2$.

theories [Eqs. (3.11) and (3.14), respectively]. The generalized mean field theory predictions were evaluated using a Padé approximant expression ${ }^{19}$ for the hard-disk monomer equation of state. As expected, the $F$ lattice formula severely overestimates the insertion probability at moderate and high densities. The lattice FH formula (not plotted) is in somewhat worse agreement with the simulation data since, as is evident from Eq. (2.23), $p_{\mathrm{FH}}<p_{\mathrm{F}}$ for $n>1$. The generalized $F$ formula underestimates the insertion probability, but does predict its order of magnitude correctly. Evidently the generalized FH formula is quite accurate at moderate and high densities, although it tends to overestimate the insertion probability as the density increases. The relation between the generalized $\mathrm{F}$ and $\mathrm{FH}$ theories and the simulation data in the present study is very similar to the relation between the lattice $F$ and $F H$ theories and simulation data for lattice chains. ${ }^{11}$ In each case, the $F$ approximation consistently underestimates the insertion probability, while FH theory yields an underestimate at low densities and an overestimate at high densities.

An important aspect of Fig. 2 is the evident smoothness 
of $\ln p(\eta, n)$ as a function of $\ln (1-\eta)$. An earlier analysis ${ }^{10}$ of Okamoto's data ${ }^{17}$ (less extensive than ours), suggested that $d \ln p(\eta, n) / d \ln (1-\eta)$ suffers a discontinuity at low density $(\eta \sim 0.2$ for $n=5)$. We have already shown ${ }^{11}$ that the insertion probability is a smooth function of density in systems of chains on the square lattice, and it is now clear that the same conclusion must be drawn for athermal chains in continuous space. One expects hard-disk chains to undergo a fluid-solid transition at the high volume fraction, but our studies were limited to the fluid phase, owing to the inefficiency of the test-chain insertion method at high volume fractions.

In Fig. 3 we compare the predictions of our generalized $F$ and $F H$ theories, and of the $F$ lattice formula with simulation results for the compressibility factor of a system of chains composed of five freely jointed, nonoverlapping tangent disks. The Monte Carlo estimate for the compressibility factor was derived using the $L=20$ data for the insertion probability. Equation (3.2) was integrated numerically, with linear interpolation of the Monte Carlo estimates for $p(\eta, 5)$. The numerical estimates for the pressure are therefore plotted as a continuous curve. Based on the uncertainties in the values for the insertion probability, we estimate the statistical uncertainty in the compressibility factor as $1 \%$ or less for $\eta \leqslant 0.45$, increasing to about $3 \%$ at $\eta=0.53$.

It is evident that the generalized FH theory provides a rather accurate prediction for the pressure. The generalized $\mathrm{F}$ theory yields a substantial overestimate for the pressure, but is far superior to the F lattice formula, Eq. (3.4). The FH lattice formula (not plotted) predicts a slightly lower pressure than that given by the $\mathrm{F}$ lattice theory. Also plotted in Fig. 3 are the results of a simulation by Okamoto ${ }^{17}$ of a system of hard-disk chains $(n=5)$ in which the angles between successive bonds are restricted to the values $\pm \pi / 2, \pi$. Apparently this constraint on internal conformations has very

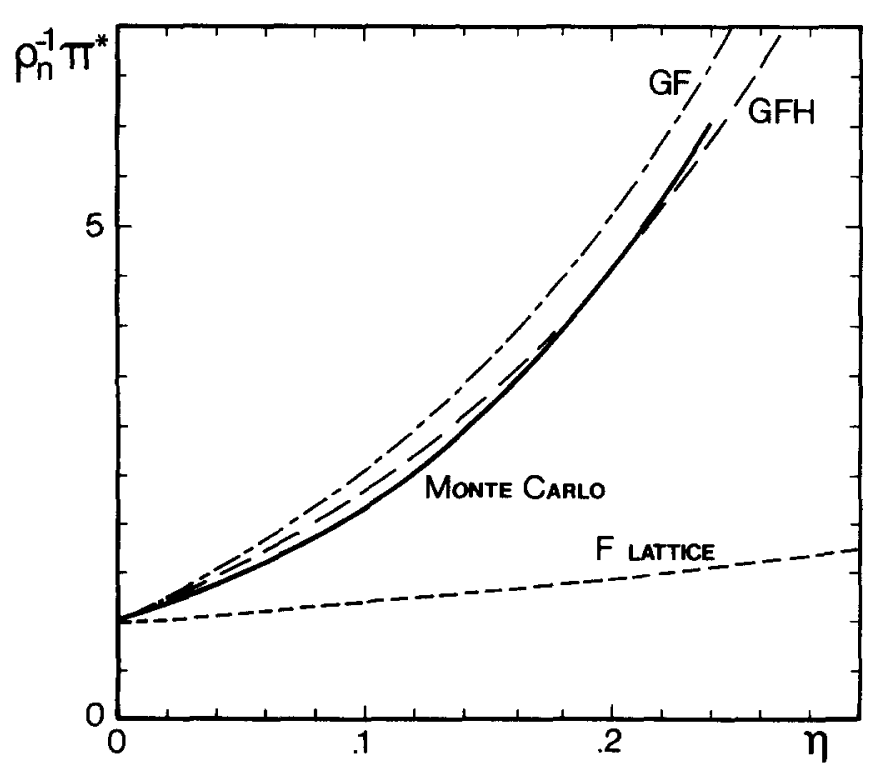

FIG. 4. Comparison of the compressibility factor $\rho_{n}^{-1} \pi^{*}$, for chains of 4 hard spheres, as obtained from Monte Carlo simulations, with predictions of the Flory lattice formula, and generalized Flory (GF) and Flory-Huggins (GFH) theories. little effect on the pressure. (Insensitivity of the pressure to the intramolecular potential is in fact predicted by $\mathrm{F}$ and $\mathrm{FH}$ theories, since the insertion probability is taken to be independent of chain configuration.)

Preliminary results of Monte Carlo simulations of three-dimensional, freely jointed tangent hard sphere chains $(n=4)$ are presented in Fig. 4. The numerical estimates were obtained via the test-chain insertion method described above, using a periodic cell of length $L=10 \sigma$. (For chains composed of $n$ tangent hard spheres of unit diameter, $\eta=n \pi N_{p} / 6 V=0.74 \rho / \rho_{c p}$, where $V$ is the volume and $\rho_{c p}=1.4133$ is the close-packing monomer density.) Due to the rapid decay of $p(\eta, n)$ with density, it is difficult to obtain accurate estimates of this quantity for $\eta>0.24$. (The statistical uncertainty in the compressibility factor is $1 \%$ or less for $\eta \leqslant 0.18$, increasing to $4 \%$ at $\eta=0.24$.) The meanfield theory predictions plotted in Fig. 4 were obtained using the Carnahan-Starling formula for the hard-sphere monomer equation of state. As in the two-dimensional example, the generalized $\mathrm{FH}$ prediction for the pressure is in excellent agreement with the simulation data. The discrepancy between the lattice-based prediction and the numerical data is even greater than in the two-dimensional case.

In view of the uncontrolled approximations invoked in the derivations, the agreement between the generalized mean field theories and the simulation data is perhaps surprising. Since the $n$-mer fluid has been treated on the basis of the monomer equation of state, this agreement suggests that the structures of the $n$-mer and monomer fluids are rather similar. (Conversely, the agreement between theory and simulation is poorest at low density, where the $n$-mer and monomer fluids are quite dissimilar.) It is of interest to determine whether the mean field theories continue to provide an accurate prediction for the equation of state at higher densities and for longer chains. Simulations bearing on these questions are in progress.

\section{ACKNOWLEDGMENTS}

The authors gratefully acknowledge the Gas Research Institute under Grant No. 5082-260-0724, and of the Donors of the Petroleum Research Fund, administered by the American Chemical Society, for the support of this work.

\section{APPENDIX: EVALUATION OF EXCLUSION VOLUMES}

Consider chains of freely jointed, nonoverlapping tangent hard sphere monomers of diameter $\sigma$. The region excluded by a chain is the union of the spheres, of radius $\sigma$, centered on each monomer. If we denote the exclusion volume of an $n$-mer (averaged over all $n$-mer configurations) by $v_{e}(n)$, then $v_{e}(1)=4 \pi \sigma^{3} / 3$, and for a dimer

$$
v_{e}(2)=2 v_{e}(1)-v_{12}(\sigma),
$$

where $v_{12}(r)$ is the volume of intersection of two circles of radius $\sigma$, whose centers are separated by a distance $r$. From elementary considerations

$v_{12}(r)=2 \pi \sigma^{3}\left[\frac{2}{3}-\frac{r}{2 \sigma}+\frac{1}{3}\left(\frac{r}{2 \sigma}\right)^{3}\right] \quad(r<2 \sigma)$,

which immediately yields Eq. (3.12). 
Now consider a trimer, with monomers centered at $r_{1}=(0,0,0), r_{2}=(0,0, \sigma)$, and $r_{3}$, with $\left|r_{3}\right|=\sigma$, and $\left|r_{2}-r_{3}\right| \geqslant \sigma$. The volume of the associated exclusion region is

$$
\begin{aligned}
v_{e}\left(r_{1}, r_{2}, r_{3}\right)= & 3 v_{e}(1)-v_{12}\left(r_{12}\right)-v_{12}\left(r_{13}\right) \\
& -v_{12}\left(r_{23}\right)+v_{123},
\end{aligned}
$$

where $r_{i j}=\left|r_{i}-r_{j}\right|$, and $v_{123}$ is the volume of intersection of the three spheres. Let $\theta$ be the angle between $r_{3}$ and the $z$ axis. If we note that $r_{12}=r_{13}=\sigma$, and that $v_{12}\left(r_{23}\right)=v_{123}$ for $\theta>2 \pi / 3$, then the average exclusion volume for a trimer may be written as

$$
\begin{aligned}
v_{e}(3)= & 3 v_{e}(1)-2 v_{12}(\sigma) \\
& -\frac{2}{3} \int_{\pi / 3}^{2 \pi / 3} d \theta \sin \theta\left[v_{12}\left(r_{23}\right)-v_{123}\right] .
\end{aligned}
$$

Since $r_{23}=2 \sigma \sin (\theta / 2)$, the integral over $v_{12}\left(r_{23}\right)$ may be evaluated using $\mathrm{Eq}$. (A2). For a given $\theta$, the region of triple intersection extends from $z=0$ to $z=(\sigma / 2)[\cos \theta+\sqrt{3}$ $\sin \theta$ ]. In a plane of fixed $z$ this region is delimited by the intersection of two circles, centered at $R_{1}=(\sigma \sin \theta, 0)$ and $R_{2}=(0,0)$, with radii

$$
\sigma_{1}=\left[\sigma^{2}-(\sigma \cos \theta-z)^{2}\right]^{1 / 2}
$$

and

$$
\sigma_{2}= \begin{cases}{\left[2 \sigma z-z^{2}\right]^{1 / 2},} & z<\frac{\sigma}{2} \\ {\left[\sigma^{2}-z^{2}\right]^{1 / 2},} & z>\frac{\sigma}{2} .\end{cases}
$$

Using this information, the integral over the three-sphere overlap volume may be evaluated numerically, yielding

$$
\int_{\pi / 3}^{2 \pi / 3} d \theta \sin \theta v_{123}=0.09775 \sigma^{3}
$$

Collecting results, one then finds

$$
\begin{aligned}
v_{e}(3) & =2 v_{e}(2)-v_{e}(1)-0.30713 \sigma^{3} \\
& =9.64125 \sigma^{3} .
\end{aligned}
$$

The last term is only about $3 \%$ of the total trimer exclusion volume. It therefore appears that for $n \geqslant 4$, the error in writing

$$
v_{e}(n) \cong v_{e}(3)+(n-3)\left[v_{e}(3)-v_{e}(2)\right]
$$

is at most a few percent. Given the approximate nature of the theories under consideration, a more precise evaluation of $v_{e}(n)$ is not warranted, and we shall employ Eq. (A7) for $n \geqslant 4$.

For chains of freely jointed, nonoverlapping tangent disks, the exclusion volumes may be evaluated in a similar manner, yielding $v_{e}(1)=\pi \sigma^{2}, v_{e}(2)=5.05482 \sigma^{2}$, and $v_{e}(3)=6.5334 \sigma^{2}$, and we again employ the approximate relation Eq. (A7) for $n \geqslant 4$.

'C. G. Gray and K. E. Gubbins, Theory of Molecular Fluids (Clarendon, Oxford, 1984), Vol. 1.

${ }^{2}$ P. J. Flory, J. Chem. Phys. 9, 660 (1941).

${ }^{3}$ M. L. Huggins, J. Chem. Phys. 9, 440 (1941).

${ }^{4} \mathrm{P}$. J. Flory, Principles of Polymer Chemistry (Cornell University, Ithaca, 1953), Chap. XII.

${ }^{5}$ H. Tompa, Polymer Solutions (Butterworth, Washington, D.C., 1956).

${ }^{6}$ P.-G. de Gennes, Scaling Concepts in Polymer Physics (Cornell University, Ithaca, 1979), Chap. III.

${ }^{7}$ E. A. Gugginheim, Applications of Statistical Mechanics (Clarendon, Oxford, 1966), Chap. 7.

${ }^{8}$ A. Bellemans and E. De Vos, J. Polym. Sci. Symp. No. 42, 1195 (1973).

${ }^{9}$ H. Okamoto and A. Bellemans, J. Phys. Soc. Jpn. 47, 955 (1979).

${ }^{10}$ A. Kolinsky, J. Polym. Sci. Polym. Lett. 22, 407 (1984).

${ }^{11}$ R. Dickman and C. K. Hall, J. Chem. Phys. 85, 3023 (1986).

${ }^{12}$ C. A. Croxton, J. Phys. A 12, 2497 (1979).

${ }^{13}$ N. F. Carnahan and K. E. Starling, J. Chem. Phys. 51, 635 (1969).

${ }^{14}$ L. Tonks, Phys. Rev. 50, 955 (1936).

${ }^{15 J}$ J. D. Bernal and J. Mason, Nature (London) 188, 910 (1960); G. D. Scott, ibid. 194, 956 (1962).

${ }^{16}$ A. J. Walton and A. G. Woodruff, Contemp. Phys. 10, 59 (1969).

${ }^{17}$ H. Okamoto, J. Chem. Phys. 64, 2686 (1975).

${ }^{18}$ N. Metropolis, A. W. Rosenbluth, M. N. Rosenbluth, A. H. Teller, and E. Teller, J. Chem. Phys. 21, 1087 (1953).

${ }^{19}$ F. H. Ree and W. G. Hoover, J. Chem. Phys. 40, 939 (1964) 